\title{
Photometric and spectroscopic study of $\mathrm{cD}$ galaxies
}

\author{
S. N. Kemp ${ }^{1}$, Ernesto Pérez-Hernández ${ }^{1}$ \\ and Víctor Hugo Ramírez-Siordia ${ }^{1,2}$ \\ ${ }^{1}$ Instituto de Astronomía y Meteorología, Universidad de Guadalajara, Av. Vallarta 2602, Col. \\ Arcos Vallarta, 44130, Guadalajara, Jalisco, México \\ email: snk@astro.iam.udg.mx \\ ${ }^{2}$ Centro de Radioastronomía y Astrofísica, Universidad Nacional Autónoma de México, \\ Campus Morelia, Apartado Postal 3-72, 58090, Morelia, Michoacán, México
}

\begin{abstract}
We have carried out photometry and spectroscopy on a sample of $10 \mathrm{cD}$ galaxies. The photometry shows, in general, fairly flat and red profile colours, implying an envelope with the same stellar population as the central galaxy. This may indicate a possible primordial origin for both structures, consistent with ideas of downsizing. Preliminary spectroscopic results are generally in agreement with the photometry, with for example younger populations at large radii for A2199, but A2589 has only younger populations.
\end{abstract}

Keywords. galaxies: evolution, galaxies: formation, galaxies: stellar content

$\mathrm{cD}$ galaxies are supergiant elliptical galaxies found usually in the centres of rich clusters. They have an extended halo-like component (envelope) and an underlying de Vaucouleurs-Sérsic elliptical galaxy-like component. The envelope can reach radial distances of $<500 \mathrm{kpc}$ (Oemler 1976, Schombert 1988). There have been many theories to explain the formation of these envelopes. These include tidal stripping (Gallagher \& Ostriker 1972), where material is stripped from neighbouring galaxies in the cluster, falling into the gravitational potential of the $\mathrm{cD}$; mergers where the envelope is built up hierarchically by successive major and minor mergers with cluster galaxies; primordial origin (Merritt 1984): where the envelope is formed simultaneously with the rest of the elliptical galaxy (which appears to be related to recent theories of "downsizing" and early formation of the largest galaxies); and cooling flows (Fabian et al. 1982): in clusters with $\mathrm{X}$-ray emission from hot gas there is frequently a minimum in $\mathrm{T}$ in the centre which can be interpreted as a flow of cooling gas towards the central regions, and if the gas cools sufficiently it could form stars, and hence the envelope populations.

The colours of the stars in the envelopes will be affected by their process of formation and subsequent evolution. We carried out a programme of deep surface photometry on a sample of $10 \mathrm{cD}$ envelopes using the $2.1 \mathrm{~m}$ and $1.5 \mathrm{~m}$ telescopes at San Pedro Mártir, Baja California, México, obtaining 30-90 min total exposure per filter. For the majority of the galaxies we obtained very flat colour profiles within the errors, varying by no more than +/- 0.1 mag in $B-V$ (Kemp et al. 2009, Guzmán Jiménez 2006, Ramírez-Siordia 2009). The profiles of A193, A496, A2634, A2589, A2670 do not show appreciable gradients. About $30 \%$ of the galaxies do have gradients, generally with bluer centres, implying recent star formation activity, possibly as a result of mergers (A2199) or cooling flows (A426). The flat profiles imply similar stellar populations in the underlying galaxy and the envelope, maybe formed at the same time. This could favour the primordial origin or downsizing hypothesis, and implies that any subsequent evolution that the galaxy experiences does not usually dominate its global colours. 


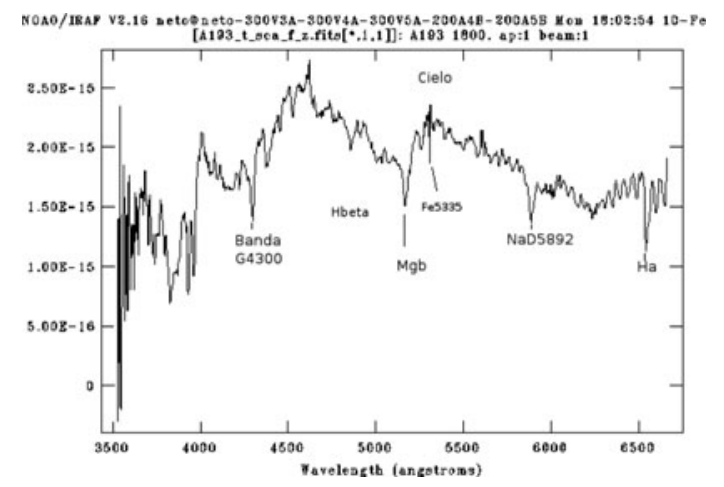

Figure 1. Spectrum of the centre of the cD galaxy in Abell 193

Variations in age and metallicity of the stellar populations with position could produce similar colours. We are carrying out a programme of medium-resolution spectroscopy of cDs at the $2.1 \mathrm{~m}$ in San Pedro Mártir and with OSIRIS/GTC to obtain age and metallicity of stellar populations. We obtained spectra of the central regions of each galaxy and various radial positions along the slit up to $r_{\mathrm{e}}$ (Pérez-Hernández 2014). We compare the central spectra of A193, A2199 (both with multiple nuclei possibly indicating mergers) and A496, A2589 (both no evidence of multiple nuclei or mergers). A193 (Fig 1) and A496 both have populations of 10-12 Gyr and metallicities near solar, compatible with their flat, red photometric colour profiles, while A2589 has young populations 26 Gyr in all positions with metallicity of $0.3-0.4$, in spite of also having a flat, red, colour profile. A2199 has younger ages at larger radii corresponding to the bluer colours seen in the photometry, and slightly sub-solar metallicities. There seem to be no major differences between galaxies with or without multiple nuclei, other factors have greater effects.

We are also carrying out analysis in 2 dimensions of galaxy profiles using GALFIT to fit components to the underlying galaxy and envelopes. The main result so far is that the extended halo component frequently has a Sérsic index of close to 1, i.e. an exponential fall off with radius.

\section{Acknowledgements}

We wish to thank CONACyT for funding the Basic Science project 'Stellar populations in early-type galaxies' which included grants for VHRS and EPH. SNK wishes to thank the PROSNI programme of the University of Guadalajara for assistance in attending this conference.

\section{References}

Fabian, A. C., Nulsen, P. E. J.., \& Canizares, C. R. 1982, MNRAS, 201, 933

Gallagher, J. S. \& Ostriker, J. P. 1972, AJ, 77, 288

Guzmán Jiménez, V. 2006, Estudio fotométrico de los colores de las envolventes de una muestra de galaxias $c D$, Masters thesis, University of Guadalajara

Kemp, S. N., Guzmán Jiménez, V., \& Ramírez Beraud, P., et al. 2009, New Quests in Stellar Astrophysics II: Ultraviolet Properties of Evolved Stellar Populations, Springer, 91

Merritt, D. 1984, ApJ, 276, 26

Oemler, A. Jr. 1976, ApJ, 209, 693 
Pérez-Hernández, E. 2014, Espectroscopía de Halos de Galaxias tipo cD's, Undergraduate thesis, University of Guadalajara

Ramírez-Siordia, V. H. 2009, Poblaciones Estelares según los colores ópticos en Galaxias Gigantes Elípticas tipo $c D$, Undergraduate thesis, University of Guadalajara

Schombert, J. M. 1988, ApJ, 328, 475 